\title{
Exponential Stability of the Genetic Regulatory Networks with Delay on Time Scales
}

\author{
Boyu Wang ${ }^{1}$, Yaru Zhao ${ }^{1}$, Jiamin $\mathrm{Li}^{1}$, Jinxiang Cai ${ }^{1}$, Honghua Bin ${ }^{1}$, Zhenkun Huang, \\ ${ }^{1}$ School of Siences, Jimei University, Xiamen 361021, China \\ ${ }^{*}$ Corresponding author
}

\begin{abstract}
Genetic regulatory networks with delay on time scales is considered in this paper. Some sufficient conditions are obtained to ensure the existence and exponential stability of a unique equilibrium of Genetic regulatory networks. The approaches are based on constructing Lyapunov functionals, the theory of calculus on time scales and the well-known Brouwer's fixed point theorem. The obtained results are general and can be applied to corresponding continuous-time and discrete-time genetic regulatory network.
\end{abstract}

Keywords- genetic regulatory networks; exponential stability; fixed point theorem; on time scales

\section{INTRODUCTION}

Genetic regulatory networks is a combination of a great number of genes and gene products interacted directly or indirectly with each other in living cells which make up a dynamic networked complex system. The dynamic behaviors of the genetic regulatory networks in living organisms become an important new area of research and received increasing attention over past few years [1], [2], [3], [4] and [10]. In addition, time delays are unavoidable in the actual evolution of the gene system, especially in the transcription and translation process. It is well-known that time delays may result in oscillation and instability of the genetic regulatory networks system. Therefore, several important results for genetic regulatory networks with time-delays have been reported in the existing literatures [5], [6]. Robust exponential stability for a class of stochastic genetic networks with uncertain parameters has been reported in [7]. According to literature [8], we know the gene interactions are characterized either in a discrete-time form or in a continuous-time case, and the topologies of the gene networks are described either deterministically or fully stochastically.Recently, the exponential stability of continuoustime and discrete-time cellular neural networks with delays has been considered in [9]. However, to the best of our knowledge, there are few investigations dealing with the stability analysis of genetic regulatory networks with delay on time scales in the existing literature. It is significant to study the genetic regulatory networks on time scales [11] which can unify the continuous and discrete situations.

Motivated by recent results [12], [13], we consider the following differential genetic regulatory networks model with delay on time scale:

$$
\left\{\begin{array}{l}
m_{i}^{\Delta}(t)=-a_{i} m_{i}(t)+\sum_{j=1}^{n} \omega_{i j} g_{j}\left(p_{j}(t-h)\right)+u_{i}, \\
p_{i}^{\Delta}(t)=-c_{i} p_{i}(t)+d_{i} m_{i}(t-\tau), i=1,2, \cdots, n .
\end{array}\right.
$$

$t \in \mathrm{T}$, where $m_{i}(t), p_{i}(t)$ are the concentrations of mRNA and protein of the ith gene at the time $t$, respectively. The parameters $a_{i}>0, c_{i}>0$ denote, respectively, the degradation rates of the mRNA and the protein. $d_{i}$ is the translation rate. If transcription factor $j$ is an activator of gene $i, \quad w_{i j}=\alpha_{i j}$; if there is no link from node $j$ to node $i, w_{i j}=0$; if transcription factor $j$ is a repressor of gene $i, w_{i j}=-\alpha_{i j}$. Where $\alpha_{i j}>0$ is a bounded constant representing the dimensionless transcriptional rate of the transcription factor $j$ to the ith gene. $u_{i}$ is defined as a basal rate, $u_{i}=\sum_{j \in V_{i 1}} \alpha_{i j}$ and $V_{i 1}$ is the set of all the $j$ which is a repressor of gene $i$. The nonlinear function $g_{j}(\cdot): \mathbb{R} \rightarrow \mathbb{R}$ represents the feedback regulation of the protein in the transcription process with $g_{j}(x)=\left(x / \beta_{j}\right)^{H_{j}} /\left(1+\left(x / \beta_{j}\right)^{H_{j}}\right), H_{j}$ where is the hill coefficient, $\beta_{j}$ is a positive constant.

\section{PRELIMINARIES}

Definition 1: A time scale $\mathrm{T}$ is an arbitrary nonempty closed subset of $\mathbb{R}$. The forward and backward jump operators $\sigma, \rho: \mathrm{T} \rightarrow \mathrm{T}$ and the graininess $\mu: \mathrm{T} \rightarrow \mathbb{R}^{+}$are defined, respectively, by $\sigma(t):=\inf \{s \in \mathrm{T}: s>t\}$,

$$
\rho(t):=\sup \{s \in \mathrm{T}: s<t\}, \mu(t):=\sigma(t)-t
$$

Definition 2: These jump operators enable us to classify the point $t$ of a time scale as right-dense, right-scattered, left- 
dense, left-scattered depending on whether $\sigma(\mathrm{t})=\mathrm{t}, \sigma(\mathrm{t})>\mathrm{t}$, $\rho(\mathrm{t})=\mathrm{t}$ and $\rho(\mathrm{t})<\mathrm{t}$, respectively for any $t \in \mathrm{T}$.

Definition 3: (Lakshmikantham and Vatsala [16]). For each $t \in \mathrm{T}$, let $\mathrm{N}$ be a neighborhood of $t$. Then, we define the generalized derivative (or Dini derivative), $D^{+} u^{\Delta}(t)$ to mean that, give $\varepsilon>0$, there exists a right neighborhood $N(\varepsilon)$ $\subset N$ of $t$ such that

$$
\frac{u(\sigma(t))-u(s)}{u(t, s)}<D^{+} u^{\Delta}(t)+\varepsilon
$$

for each $s \in N(\varepsilon), s>t$, where $u(t, s)=\sigma(t)-s$. gets

In case $t$ is right-scattered and $\mathrm{u}(\mathrm{t})$ is continuous at $t$, one

$$
D^{+} u^{\Delta}(t)=\frac{u(\sigma(t))-u(t)}{\sigma(t)-t}
$$

For simplicity, we denote $C_{r d}$ by the set of all right-dense continuous functions. If $p(t) \in C_{r d}$ and $1+\mu(t) p(t)>0$, then $p(t)$ is said to be a positive regressive function. Denote $R^{+}$by the set of all regressive function. Next we give the definition of the exponential function and list its useful properties.

Definition 4: (Bohner and Peterson [14]). If $p \in \mathfrak{R}^{+}$is a regressive function, then the generalized exponential function $e_{p}(t, s)$ is defined by

$$
e_{p}(t, s)=\exp \left\{\int_{s}^{t} \xi_{\mu(\tau)}(p(\tau)) \Delta \tau\right\}, s, t \in \mathrm{T},
$$

with the cylinder transformation

$$
\xi_{h}(z)= \begin{cases}\frac{\log (1+h z)}{h}, & h \neq 0 \\ z, h=0, & h=0\end{cases}
$$

Definition 5: The equilibrium $\left(m^{*}, p_{i}^{*}\right)=\left(m_{1}^{*}, \cdots, m_{h}^{*}, p_{1}^{*}, \cdots p_{n}^{*}\right)$ of system (1) is said to be exponentially stable if there exist a $\xi>0, p \in R^{+}$and $\Gamma=\Gamma(p)>0$ such that every solution $\left(m_{1}, \cdots, m_{n}, p_{1} \cdots p_{n}\right)$ of system (1) satisfying

$$
\sum_{i=1}^{n}\left|m_{i}(t)-m_{i}^{*}\right|+\sum_{i=1}^{n}\left|p_{i}(t)-p_{i}^{*}\right| \leq \frac{\Gamma(p)}{\xi e_{p}(t, 0)}
$$

$\times\left(\sum_{i=1}^{n} \sup _{s \in[-\tau, 0]_{]}}\left|m_{i}(s)-m_{i}^{*}\right|+\sum_{i=1}^{n} \sup _{s \in[-h, 0]_{\square}}\left|p_{i}(s)-p_{i}^{*}\right|\right), t \in \mathrm{T}_{0}^{+}$.

Lemma 1: (Bohner and Peterson [15]) If $p, q \in R$, then

(i) $e_{p}(\sigma(t), s)=(1+\mu(t) p(t)) e_{p}(t, s)$;

(ii) $\frac{1}{e_{p}(t, s)}=e_{\Theta p}(t, s)$, where $e_{\Theta p}(t, s)=-\frac{p(t)}{1+\mu(t) p(t)}$;

(iii) $e_{p}(t, s) e_{p}(s, r)=e_{p}(t, r)$;

(iv) $e_{p}(t, s) e_{q}(t, s)=e_{p \oplus q}(t, s)$;

(v) $\frac{e_{p}(t, s)}{e_{\mathrm{q}}(t, s)}=e_{p \oplus q}(t, s)$;

(vi) $\left(\frac{1}{e_{p}(\cdot, s)}\right)^{\Delta}=-\frac{p(t)}{e_{p}{ }^{\sigma}(\cdot, s)}$; where $\mathfrak{R}:=\left\{p \in C_{r d}: 1+\mu(t) p(t) \neq 0\right\}$.

Finally, we state Brouwer's fixed point theorem which enables us to prove the existence of a unique equilibrium of (1).

Theorem 1: If $K \in \mathbb{R}^{n}$ is a bounded closed convex set.

$\Xi \in C(K, K)$, then there exists a $x^{*} \in K$ with $\Xi x^{*}=x^{*}$.

Throughout this paper, we make basic assumption as follows:

$$
\begin{aligned}
& \left(H_{1}\right): g_{i}: \mathbb{R} \rightarrow \mathbb{R},\left|g_{i}(x)-g_{i}(y)\right| \leq L_{i}|x-y|, \\
& \qquad\left|g_{i}(x)\right| \leq M_{i}, \text { for all } x, y \in \mathbb{R} . \\
& \text { III. EXISTENCE AND UNIQUENESS OF A EQUILIBRIUM }
\end{aligned}
$$

\section{EXISTENCE AND UNIQUENESS OF A EQUILIBRIUM}

Theorem 2: Suppose $\left(H_{1}\right)$ hold, then system (1) has a unique equilibrium state if

$$
\sum_{j=1}^{n} \frac{\left|d_{i}\right|}{a_{i} c_{i}}\left|w_{j i}\right| L_{i}<1, i=1,2, \cdots, n
$$


Proof: An equilibrium $\left(m_{1}{ }^{*}, m_{2}{ }^{*}, \cdots, m_{n}{ }^{*}, p_{1}{ }^{*}, p_{2}{ }^{*}, \cdots p_{n}{ }^{*}\right)$ of (1) is a solution of the system

$$
\left\{\begin{array}{l}
-a_{i} m_{i}^{*}+\sum_{j=1}^{n} \omega_{i j} g_{j}\left(p_{j}^{*}\right)+u_{i}=0, \\
-c_{i} p_{i}^{*}+d_{i} m_{i}^{*}=0, i=1,2, \ldots, n,
\end{array}\right.
$$

which leads to

$$
p_{i}^{*}=\frac{d_{i}}{a_{i} c_{i}}\left[\sum_{j=1}^{n} w_{i j} g_{j}\left(p_{j}^{*}\right)+u_{i}\right], i=1,2, \cdots, n .
$$

Due to the boundedness of the activations, we have

$$
\left|\frac{d_{i}}{a_{i} c_{i}}\left(\sum_{j=1}^{n} w_{i j} g_{j}\left(p_{j}^{*}\right)+u_{i}\right)\right| \leq \frac{\left|d_{i}\right|}{a_{i} c_{i}}\left(\sum_{j=1}^{n}\left|w_{i j}\right| M_{j}+\left|u_{i}\right|\right)=A_{i} .
$$

Define a function $h: \mathbb{R}^{n} \rightarrow \mathbb{R}^{n}$ as follows:

$$
\begin{gathered}
h\left(x_{1}, x_{2}, \cdots, x_{n}\right)=\left\{h_{1}\left(x_{1}, x_{2}, \cdots, x_{n}\right), \cdots, h_{n}\left(x_{1}, x_{2}, \cdots, x_{n}\right)\right\}, \\
h\left(x_{1}, x_{2}, \cdots, x_{n}\right)=\frac{d_{i}}{a_{i} c_{i}}\left[\sum_{j=1}^{n} w_{i j} g_{j}\left(x_{j}^{*}\right)+u_{i}\right] .
\end{gathered}
$$

Then it will follow that $h=\left(h_{1}, h_{2}, \cdots, h_{n}\right)$ maps the bounded closed convex set $D=D_{1} \times D_{2} \times \cdots \times D_{n}$ into itself where $D_{i}=\left[-A_{i}, A_{i}\right]$. By Brouwer's fixed point theorem, there exists a fixed points say $p^{*}$ of $\mathrm{h}$ such that $p^{*}=h\left(p^{*}\right)$ or equivalently

$$
p_{i}^{*}=h_{i}\left(p_{1}^{*}, p_{2}^{*}, \cdots, p_{n}^{*}\right)=\frac{d_{i}}{a_{i} c_{i}}\left[\sum_{j=1}^{n} w_{i j} g_{j}\left(p_{j}^{*}\right)+u_{i}\right],
$$

we can now define $m_{i}^{*}=\frac{c_{i}}{d_{i}} p_{i}^{*}, i=1,2, \cdots n$, so that $\left(m_{1}{ }^{*}, m_{2}{ }^{*}, \cdots, m_{n}{ }^{*}, p_{1}{ }^{*}, p_{2}{ }^{*}, \cdots p_{n}{ }^{*}\right)$ is an equilibrium of (1).

Suppose that there are two equilibrium solutions of (1) say $\quad\left(m_{1}{ }^{*}, \quad m_{2}{ }^{*}, \cdots, m_{n}{ }^{*}, p_{1}{ }^{*}, p_{2}{ }^{*}, \cdots p_{n}{ }^{*}\right) \quad$ and $\left(\bar{m}_{1}^{*}, \bar{m}_{2}^{*}, \cdots, \bar{m}_{n}^{*}, \bar{p}_{1}^{*}, \bar{p}_{2}^{*}, \cdots \bar{p}_{n}^{*}\right)$. Then we have

$$
p_{i}^{*}-\bar{p}_{i}^{*}=\sum_{j=1}^{n} \frac{d_{i}}{a_{i} c_{i}} w_{i j}\left[g_{j}\left(p_{j}^{*}\right)-g_{j}\left(\bar{p}_{j}^{*}\right)\right], i=1,2, \cdots, n .
$$

Which leads to

$$
\sum_{i=1}^{n}\left|p_{i}^{*}-\bar{p}_{i}^{*}\right| \leq \sum_{i=1}^{n} \sum_{j=1}^{n} \frac{\left|d_{i}\right|}{a_{i} c_{i}}\left|w_{i j}\right| L_{j}\left|p_{j}^{*}-\bar{p}_{j}^{*}\right|, i=1,2, \cdots, n .
$$

So we have

$$
\sum_{i=1}^{n}\left[1-\sum_{j=1}^{n} \frac{\left|d_{i}\right|}{a_{i} c_{i}}\left|w_{j i}\right| L_{i}\right]\left|p_{i}^{*}-\bar{p}_{i}^{*}\right| \leq 0, i=1,2, \cdots, n .
$$

(2) implies that $p_{i}^{*}=\bar{p}_{i}^{*}, i=1,2, \cdots, n$. And we have $m_{i}^{*}$ $=\bar{m}_{i}^{*}, i=1,2, \cdots, n$. The proof is completed.

\section{EXPONENTIAL STABILITY OF THE EQUILIBRIUM}

Let $\left(m_{1}{ }^{*}, m_{2}{ }^{*}, \cdots, m_{n}{ }^{*}, p_{1}{ }^{*}, p_{2}{ }^{*}, \cdots p_{n}{ }^{*}\right)$ be the equilibrium of the system (1). Let $x_{i}(t)=m_{i}(t)-m_{i}^{*}, y_{i}(t)=p_{i}(t)-p_{i}^{*}, i=1,2, \cdots, n$.

Then we can rewrite the system (1) as

$$
\left\{\begin{array}{l}
x_{i}^{\Delta}(t)=-a_{i} x_{i}(t)+\sum_{j=1}^{n} \omega_{i j}\left[g_{j}\left(y_{j}(t-h)+p_{j}^{*}\right)-g_{j}\left(p_{j}^{*}\right)\right], \\
y_{i}^{\Delta}(t)=-c_{i} y_{i}(t)+d_{i} x_{i}(t-\tau), i=1,2, \ldots, n,
\end{array}\right.
$$

for all $t \in \mathrm{T}_{0}^{+}$.

It is thus sufficient to establish the stability of the trivial solution of (3) in order to establish the stability of the equilibrium $\left(m_{1}{ }^{*}, m_{2}{ }^{*}, \cdots, m_{n}{ }^{*}, p_{1}{ }^{*}, p_{2}{ }^{*}, \cdots p_{n}{ }^{*}\right)$ of our original system.

Theorem 3: Assume that $\left(H_{1}\right)$ holds, suppose further that: $\left(H_{2}\right)$ : If there exist some constants $\lambda_{i}>0, \xi_{i}>0$ and $p \in \mathfrak{R}^{+}$such that

$$
\begin{gathered}
\xi_{i}\left[p-a_{i}(1+p \mu(t))\right]+\lambda_{i}\left|d_{i}\right|(1+p \mu(t+\tau)) e_{p}(t+\tau, t)<0, \\
\lambda_{i}\left[p-c_{i}(1+p \mu(t))\right]+\sum_{j=1}^{n} \xi_{j}\left|w_{j i}\right| L_{i}(1+p \mu(t+h)) e_{p}(t+h, t)<0,
\end{gathered}
$$

For all $i=1,2, \cdots, n, t \in \mathrm{T}_{0}^{+}$. Then the trivial solution of (3) is exponentially stable.

Proof: It follows the Theorem 2 that the trivial solution of (3) is a unique equilibrium of (3). Now we construct the Laypunov functional $V(t)=V_{1}(t)+V_{2}(t)+V_{3}(t)+V_{4}(t)$, where 


$$
\begin{aligned}
& V_{1}(t)=\sum_{i=1}^{n} \xi_{i} e_{p}(t, 0)\left|x_{i}(t)\right|, V_{3}(t)=\sum_{i=1}^{n} \lambda_{i} e_{p}(t, 0)\left|y_{i}(t)\right|, \\
& V_{2}(t)=\sum_{i=1}^{n} \sum_{j=1}^{n} \xi_{i}\left|w_{i j}\right| L_{j} \int_{t-h}^{t}(1+p \mu(s+h)) e_{p}(s+h, 0)\left|y_{j}(s)\right| \Delta s, \\
& V_{4}(t)=\sum_{i=1}^{n} \lambda_{i}\left|d_{i}\right| \int_{t-\tau}^{t}(1+p \mu(s+\tau)) e_{p}(s+\tau, 0)\left|x_{i}(s)\right| \Delta s .
\end{aligned}
$$

Calculating $D^{+} V(t)^{\Delta}$ along (3), we can get

$$
\begin{aligned}
& D^{+}\left(V_{1}(t)+V_{2}(t)\right)^{\Delta} \leq \sum_{i=1}^{n} \xi_{i}\left[p-a_{i}(1+p \mu(t))\right] e_{p}(t, 0)\left|x_{i}(t)\right| \\
& +\sum_{i=1}^{n} \sum_{j=1}^{n} \xi_{j}\left|w_{j i}\right| L_{i}(1+p \mu(t+h)) e_{p}(t+h, 0)\left|y_{j}(t)\right|
\end{aligned}
$$

$$
\begin{gathered}
D^{+}\left(V_{3}(t)+V_{4}(t)\right)^{\Delta} \leq \sum_{i=1}^{n} \lambda_{i}\left[p-c_{i}(1+p \mu(t))\right] e_{p}(t, 0)\left|y_{i}(t)\right| \\
\quad+\sum_{i=1}^{n} \lambda_{i}\left|d_{i}\right|(1+p \mu(t+\tau)) e_{p}(t+\tau, 0)\left|x_{i}(t)\right|
\end{gathered}
$$

From (4) and (5), we can get

$$
\begin{gathered}
D^{+} V(t)^{\Delta} \leq \sum_{i=1}^{n}\left\{\xi_{i}\left[p-a_{i}(1+p \mu(t))\right]\right. \\
\left.+\lambda_{i}\left|d_{i}\right|(1+p \mu(t+\tau)) e_{p}(t+\tau, t)\right\} e_{p}(t, 0)\left|x_{i}(t)\right| \\
+\sum_{i=1}^{n}\left\{\lambda_{i}\left[p-c_{i}(1+p \mu(t))\right]+\sum_{j=1}^{n} \xi_{j}\left|w_{j i}\right|\right. \\
\left.\times L_{i}(1+p \mu(t+h)) e_{p}(t+h, t)\right\} e_{p}(t, 0)\left|y_{i}(t)\right| .
\end{gathered}
$$

By using $\left(H_{2}\right)$, we can conclude that $V(t) \leq V(0)$ for $t \in \mathrm{T}^{+}$. On the other hand, we have

$$
V(0)=\Gamma(p)\left(\sum_{i=1}^{n} \sup _{s \in[-\tau, 0]_{\mathrm{T}}}\left|x_{i}(s)\right|+\sum_{i=1}^{n} \sup _{s \in[-h, 0]_{\mathrm{T}}}\left|y_{i}(s)\right|\right),
$$

where $\Gamma(p)=\max \left\{\Delta_{1}, \Delta_{2}\right\}$,

$$
\begin{aligned}
& \Delta_{1}=\max _{i}\left\{\xi_{i}+\lambda_{i}\left|d_{i}\right| \int_{-\tau}^{0}(1+p \mu(s+\tau)) e_{p}(s+\tau, 0) \Delta s\right\}, \\
& \Delta_{2}=\max _{i}\left\{\lambda_{i}+\sum_{i=1}^{n} \xi_{j}\left|w_{j i}\right| L_{i} \int_{-h}^{0}(1+p \mu(s+h)) e_{p}(s+h, 0) \Delta s\right\},
\end{aligned}
$$

which means that

$$
\min \left\{\xi_{i}, \lambda_{i}\right\} e_{p}(t, 0)\left(\sum_{i=1}^{n}\left|x_{i}(t)\right|+\sum_{i=1}^{n}\left|y_{i}(t)\right|\right) \leq V(t) \leq V(0)
$$

Thus, we finally get

$$
\begin{gathered}
\sum_{i=1}^{n}\left|x_{i}(t)\right|+\sum_{i=1}^{n}\left|y_{i}(t)\right| \leq \frac{\Gamma(p) e_{\Theta p}(t, 0)}{\min \left\{\xi_{i}, \lambda_{i}\right\}}\left(\sum_{i=1}^{n} \sup _{s \in[-\tau, 0]_{\mathrm{T}}}\left|x_{i}(s)\right|\right. \\
\left.+\sum_{i=1}^{n} \sup _{s \in[-h, 0]_{\mathrm{T}}}\left|y_{i}(s)\right|\right) .
\end{gathered}
$$

Therefore, the trivial solution of (3) is exponentially stable.

The proof is completed.

From Theorem 2 and Theorem 3 we can obtain the following result.

Corollary 1: Suppose $\left(H_{1}\right)$ and (2) holds, and there exist some constants $\lambda_{i}>0, \xi_{i}>0$ and $p>0$ such that

$$
\left\{\begin{array}{l}
\xi_{i}\left(p-a_{i}\right)+\lambda_{i}\left|d_{i}\right| \mathrm{e}^{p \tau}<0, \\
\lambda_{i}\left(p-c_{i}\right)+\mathrm{e}^{p h} \sum_{j=1}^{n} \xi_{j}\left|w_{j i}\right| \mathrm{L}_{i}<0, i=1,2, \ldots, n,
\end{array}\right.
$$

$t \in \mathrm{T}_{0}^{+}$. then system (1) exists a unique equilibrium which is exponentially stable.

From Theorem 2 and Theorem 3 we can also obtain the following corollary.

Remark 1: Conditions (6) can be replaced by

$$
-\xi_{i} a_{i}+\lambda_{i}\left|d_{i}\right|<0,-c_{i} \lambda_{i}+\sum_{j=1}^{n} \xi_{j}\left|w_{j i}\right| \mathrm{L}_{i}<0
$$

Remark 2: If the time scales $\mathrm{T}=\mathbb{Z}(\mu(t)=1)$ then system (1) also includes the discrete-time delayed genetic regulatory networks model as its special cases: 


$$
\left\{\begin{array}{l}
m_{i}(k+1)-m_{i}(k)=-a_{i} m_{i}(k)+u_{i}+\sum_{j=1}^{n} w_{i j} g_{j}\left(p_{j}(k-h)\right), \\
p_{i}(k+1)-p_{i}(k)=-c_{i} p_{i}(k)+d_{i} m_{i}(k-\tau), \quad i=1,2, \ldots, n,
\end{array}\right.
$$

where $k \in\{0,1, \cdots\}, h$ and $\tau$ are positive integers.

Corollary 2: Suppose $\left(H_{1}\right)$ and (2) holds, and there exist some constants $\lambda_{i}>0, \xi_{i}>0$ and $p>0$ such that

$$
\begin{gathered}
\xi_{i}\left[p-a_{i}(1+p)\right]+\lambda_{i}\left|d_{i}\right|(1+p)^{\tau+1}<0, \\
\lambda_{i}\left[p-c_{i}(1+p)\right]+(1+p)^{h+1} \sum_{j=1}^{n} \xi_{j}\left|w_{j i}\right| L_{i}<0, i=1,2, \cdots, n .
\end{gathered}
$$

$t \in \mathrm{T}_{0}^{+}$. then system (1) exists a unique equilibrium which is exponentially stable.

\section{CONCLUDING REMARKS}

In this paper, we studied exponential stability of the genetic regulatory networks model with delay on time scales an obtained some more generalized results to ensure the existence, uniqueness and global exponential stability of the equilibrium. These results can give a significant insight into the complex dynamical structure of genetic regulatory networks model. The conditions are easily checked in practice by simple algebraic methods.

\section{REFERENCE}

[1] L. Chen, K. Aihara, Stability of genetic regulatory networks with time delay, IEEE Trans. Circuits Syst, 2002, pp. 602-608.

[2] C. Li, L. Chen, K. Aihara, Synchronization of coupled nonidentical enetic oscillators, Phys. Biol, 2006, pp. 37-44.

[3] P.Balasubramaniam,R.Rakkiyappan,R. Krishnasamy,Stochastic stability of Markovian jumping uncertain stochastic genetic regulatory networks with interval time-varying delays, Math. Biosci, 2010, pp. 97-108.

[4] W.Zhang, J.Fang, Y.Tang, New robust stability analysis for genetic regulatory networks with random discrete delays and distributed delays, Neurocomputing, 2011, pp. 44-60

[5] X. Lou, Q. Ye, B. Cui, Exponential stability of genetic regulatory networks with random delays, Neurocomputing, 2010, pp. 759-769.

[6] Z. Wang, X. Liao, S. Guo, H. Wu, Mean square exponential stability of stochastic genetic regulatory networks with time-varying delays, Inf. Sci, 2011, pp. 792-811.

[7] G. Wang, J. Cao, Robust exponential stability analysis for stochastic genetic networks with uncertain parameters, Commun. Nonlinear. Sci, 2009, pp. 3369-3378.

[8] I. Ivanov, E. Dougherty, Modeling genetic regulatory networks: continuous or discrete?, J Biol. Syst, 2006, pp. 219-230.

[9] S. Mohamad, K. Gopalsamy, Exponential stability of continuous-time and discrete-time cellular neural networks with delays, Appl. Math. Comput, 2003, pp. 17-38.

[10] J. Hu, J. Liang, J. Cao, Stability analysis for genetic regulatory networks with delays: The continuous-time case and the discrete-time case, Applied Mathematics and Computation, 2013, pp. 507-517.
[11] S. Hilger, Analynis on measure chains-unified approach to continuous and discrete calculus, Results in Mathematics, 1990, pp. 18-56.

[12] S. Lakshmanana, Fathalla A. Rihan, Stability analysis of the differential genetic regulatory networks model with time-varying delays and Markovian jumping parameters, Nonlinear Analysis, Hybrid Syst, 2014, pp. 115.

[13] K.Gopalsamy, Second order neurons and learning in cohen-grossberg networks,International Joural of Neural Systems, 2006, pp. 179-189.

[14] M. Bohner, A. Peterson, Dynamic Equations on Time Scales, An introduction with applications, Birkhauser Boston, 2001.

[15] M. Bohner, A. Peterson, Advance in Dynamic Equations on Time Scales, Birkhauser Boston, 2003.

[16] V. Lakshmikantham, A. Vatsala, Hybrid systems on time scales, J. Of Comput. Appl. Math., 2002, pp. 227-235.

[17] B. Shi, X. Wang, Introductions to Functional Analysis with Application, Beijing, 2006. 